\title{
Multidimensional treatment foster care was more effective than group care for chronic juvenile offenders
}

Chamberlain P, Reid JB. Comparison of two community alternatives to incarceration for chronic juvenile offenders. J Consult Clin Psychol 1998 Aug;66:624-33.

\section{Question}

In adolescent boys with histories of serious and chronic delinquency, what is the effectiveness of a multidimensional treatment foster care (MTFC) programme compared with community group care (GC)?

\section{Design}

Randomised controlled trial with 12 months follow up.

\section{Setting}

Oregon, USA.

\section{Participants}

85 boys who were 12 to 17 years of age (mean age 15 y, 85\% white) with histories of serious and chronic delinquency (mean of 14 previous criminal referrals) and were referred for community placement by the juvenile justice system. Boys who were judged to require inpatient drug and alcohol treatment were excluded. Consent to participate was given for 79 boys.

\section{Intervention}

Participants were allocated to MTFC $(n=37)$ or GC $(n=42)$. MTFC involved living in a foster family in which the foster parents were trained in behaviour management methods to provide a structured daily living environment with close supervision, clear rules and limits, and consequences for rule infractions. MTFC participants also attended school and were monitored for attendance, homework completion, and attitude; and attended weekly family therapy sessions. Boys in GC programmes lived in group homes staffed by shift workers. Most of the GC programmes used the positive peer culture approach in which the boys participated in group work and in the establishment of discipline and decision making rules.

\section{Main outcome measures}

1 year rates of running away, incarceration, and programme completion; and rates of delinquent activity assessed by official criminal referrals and self reported delinquency (Elliott Behavior Checklist).

\section{Main results}

Fewer boys in MTFC ran away from their placements than did boys in GC $(31 \%$ v $58 \%, \mathrm{p}=0.02)$ and spent less time incarcerated either in detention or state training schools (53 v 129 mean days, $\{95 \% \text { CI for the } 76 \text { day difference } 32 \text { to } 121\}^{*}$, $\mathrm{p}=0.01)$. More boys in MTFC completed their programmes than did boys in GC $(73 \% v 36 \%, \mathrm{p}=0.001)$. Greater reductions in official criminal referrals occurred among boys in MTFC (mean decrease 5.9 criminal referrals/y) than among boys in GC (mean decrease 1.3 criminal referrals/y) \{CI for the 2.8 referral difference at 12 mo post-termination 1.0 to 4.6$\}^{*}$. For self reported delinquency, greater reductions were seen in all 3 subscales of the Elliott Behavior Checklist among boys in MTFC than boys in GC: general delinquency (12.8 $v 28.9$, \{CI for the 16.1 difference 3.8 to 28$\}^{*}, \mathrm{p}=0.01$ ), index offences $(3.2 v 8.6,\{\mathrm{CI}$ for the 5.4 difference 1.0 to 9.8$\}^{*}, \mathrm{p}=0.03$ ), and felony assaults (1.2 $v 2.7$, $\{\text { CI for the } 1.5 \text { difference } 0.004 \text { to } 3.0\}^{*}, \mathrm{p}=0.05$ ).

\section{Conclusion}

Multidimensional treatment foster care was more effective than group care in reducing criminal behaviour in adolescent boys with histories of serious and chronic delinquency.

*Numbers calculated from data in article.

Source of funding: National Institute of Mental Health.

For correspondence: Dr P Chamberlain, Oregon Social Learning Center, 160 East Fourth Avenue, Eugene, OR 97401, USA. Fax +1 5414857087.

\section{Commentary}

Despite the social and economic effects of chronic juvenile offenders, scientific studies with appropriate methodology are rare.

This well done study by Chamberlain and Reid compared 2 community based alternatives to incarceration. The elaborate MTFC programme was more effective than GC, presumably because it separated the participants from other delinquent boys and so from harmful peer influence. This approach seems reasonable given that traditional ethical and social values are best transferred in a family situation and peer influence can be a potentially destabilising factor on social behaviour in this age group.

If the foster parent-offender interaction is stable and positive, outcomes can improve, even if delinquency began early in life. This corresponds with recent psychodynamic and behavioural therapy findings that emphasise the effect of the personality of the therapist (in this case, the foster parent) rather than transference phenomena or specialised psychotherapeutic techniques. Furthermore, simply living in a family or even having irregular contact with one's own family, seems to be a protective factor concerning further juvenile crimes. ${ }^{1}$

It is unfortunate that the therapeutic effect on the participants cannot be precisely estimated because both groups received different amounts of professional help with regard to family therapy (85\% of MTFC $v 50 \%$ of GC participants) and individual therapy $(100 \%$ of MTFC $v$ $67 \%$ of GC participants).
The MTFC approach is likely to be more expensive than GC in the short term but probably not in the long term. ${ }^{2} \mathrm{~A}$ cost benefit analysis would therefore have been of great value for developing policies. However, because this study is part of a larger project, hopefully these aspects will be discussed in another interesting and stimulating paper.

Oliver Bilke, MD Medical University of Lübeck Lübeck, Germany

Alfred Behnisch, MD

Christian Albrechts - Kiel University

Kiel, Germany

1 Lewis DO, Yeager CA, Lovely R, et al.J Am Acad Child Adolesc Psychiatry 1994;33:518-28. 2 Daly DL, Dowd TP. Child Welfare 1992;71:487- 